\title{
Real time fractional-order control technique for coupled tank liquid level control process
}

\author{
Ahmet Dumlu *, Kagan Koray Ayten \\ Department of Electrical \& Electronics Engineering, College of Engineering, Erzurum Technical University, Erzurum, Turkey
}

\section{A R T I C L E IN F O}

\section{Article history:}

Received 22 February 2017

Received in revised form

10 September 2017

Accepted 25 September 2017

\section{Keywords:}

Liquid level control system

Fractional order controller

$\mathrm{PI}^{\lambda} \mathrm{D}^{\mu}$ controller

\begin{abstract}
A B S T R A C T
This paper presents real time fractional-order control technique for a coupled tank liquid level system that often used in industry. First of all system dynamic model has been presented. And then two different control approaches such as the classical proportional-integral-derivative (PID) and fractional-order $\mathrm{PI}^{\lambda} \mathrm{D}^{\mu}$ control are used to improve the tracking performance of the coupled tank liquid level system. Design procedures for both controllers are given in detail. Additionally, to verify the effectiveness of the fractional-order PID controller, detailed experimental comparisons with a feedforward proportional integral controller has been realized. It is observed that not only transient but also steady-state error values have been reduced with the aid of the $\mathrm{PI}^{\lambda} \mathrm{D}^{\mu}$ controller for tracking control purpose.
\end{abstract}

(C) 2017 The Authors. Published by IASE. This is an open access article under the CC BY-NC-ND license (http://creativecommons.org/licenses/by-nc-nd/4.0/).

\section{Introduction}

The liquid level and flow control between the two or more tanks is an important application in many industrial areas such as power generation $(\mathrm{Q}$ et al., 2011), biochemical (Boiocchi et al., 2015) and petrochemical processing, water distribution (Kaplanoglu et al., 2015) and so on. In these industries, the liquid can be stored in a tank, pumped into another tank or mixed with chemical liquids. The coupled tank system can be used in many industries that consist of two vertical tanks which connected together with an orifice and electrical pumps, motorized valve which are typical actuators in these systems. In addition, pressure sensor and flowmeter provide liquid level and rate of flow of liquid measurement for the purpose of control applications.

The coupled tank systems are nonlinear due to pump, valve characteristics and parameter variations. Therefore, the application of liquid level control in a tank and flow between tanks is a basic problem for these systems. PID controllers are used for liquid level control in most applications. However, the classical PID controllers are unable to meet the demands of high precise level control; in spite of these controllers is the perfect controller for simple and linear systems. The real systems are not

\footnotetext{
* Corresponding Author.

Email Address: ahmetdumlu@erzurum.edu.tr (A. Dumlu) https://doi.org/10.21833/ijaas.2017.011.020

2313-626X/C) 2017 The Authors. Published by IASE.

This is an open access article under the CC BY-NC-ND license

(http://creativecommons.org/licenses/by-nc-nd/4.0/)
}

exactly linear, but may be identified as linearized models around a nominal operating point. The parameters of controller are tuned at that point may not reflect the real-time system characteristics due to parameter variations. For this purpose, to perform high precision liquid level control and good tracking reference signal, it is required to use a nonlinear control method for solving above mentioned problems.

Numerous nonlinear control methods are applied to couple tank systems that have been reported in available literature. To perform high precision liquid level control under the disturbance and parameter variations adaptive based controller has been investigated (Bhuvaneswari et al., 2009). Also, a sliding mode control is an effective, robust control method for the system which included unknown parameters and un-modelled dynamics have been applied to coupled tank system (Derdiyok and Başçi, 2013; Musmade and Patre, 2013).

In classical PID control, there exist four weaknesses such as error computation; noise degradation in the derivative control; oversimplification and the loss of performance in the control law in the form of a linear weighted sum; and complications brought by the integral control.

In order to enhance the robustness and performance of PID control systems, Podlubny has proposed a generalization of the PID controllers, namely, $\mathrm{PI}^{\lambda} \mathrm{D}^{\mu}$ fractional-order controller (Podlubny, 1999a). In the proposed work, the improvement of the proposed controller has been provided by using an integrator of order $\lambda$ and a differentiator of order 
$\mu$ (the orders $\lambda$ and $\mu$ may assume real noninteger values).

Numerous design methodologies intended for $\mathrm{PI}^{\lambda} \mathrm{D}^{\mu}$ controllers are reported in the literature. Among the many applications of fractional-order controllers to engineering problems, those to a flexible belt transmission (Oustaloup et al., 1995), an active suspension system (Altet et al., 2004), irrigation canals (Domingues et al., 2010), or the control of robots (Dumlu and Erenturk, 2014) can be mentioned. In this paper, a state-coupled tanks liquid level system is introduced, and the dynamic model of the system is formulated first. And then in order to illustrate the efficiency of fractional controller, experimental study has been realized. Employing the $\mathrm{PI}^{\lambda} \mathrm{D}^{\mu}$ controller for tracking control purpose, not only transient but also steady-state error values have been reduced.

The main contribution of this paper is to determine the control action with the aid of the fractional-order $\mathrm{PI}^{\lambda} \mathrm{D}^{\mu}$ controller different from previously defined controller structures. The determination of correct and accurate control action has great importance when high accuracy needed for the trajectory tracking control of a state-coupled tanks liquid level system.

\section{System description and models}

A schematic diagram of the coupled-tank closed recirculating test system is depicted in Fig. 1. This system includes an electrically driven pump with a water reservoir and two tanks connected together with an orifice and pressure sensor for level measurement at the bottom of each tank. In this experimental setup, the two tanks are mounted on the front plate and the electrical pump feeds into Tank 1. And then the outflow of Tank 1 feeds into Tank 2. Flow from the Tank 2 flows into the main water reservoir. The liquid levels in Tanks 1 and 2 are measured by using two pressure-sensitive sensor located at the bottom of each tank.

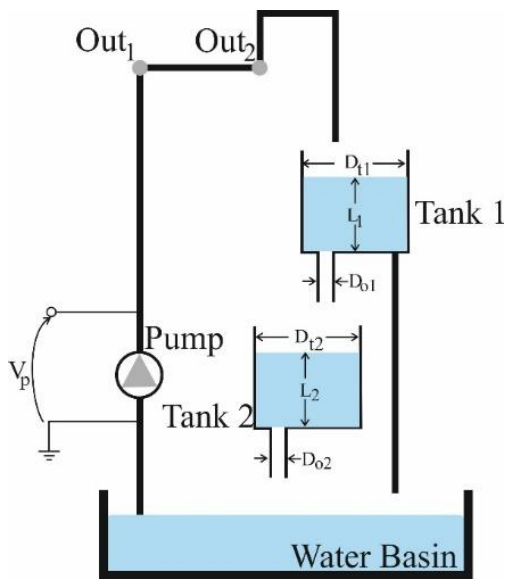

Fig. 1: Schematic diagram of the coupled-tank closed recirculating system

The time derivation of the liquid level in each tank is given as follows (Eq. 1).
$\dot{\mathrm{L}}_{\mathrm{i}}(\mathrm{t})=\frac{1}{\mathrm{~A}_{\mathrm{i}}}\left(\mathrm{F}_{\mathrm{IN}_{\mathrm{i}}}-\mathrm{F}_{\mathrm{OUT}_{\mathrm{i}}}\right) \mathrm{i}=1,2$

where $L_{i}(t)$ is the level of liquid in the tank $(\mathrm{cm}), A_{i}$ is the cross-sectional area $\left(\mathrm{cm}^{2}\right)$ and $F_{I N_{i}}, F_{O U T_{i}}$ are inflow and outflow rate $\left(\mathrm{cm}^{3} / \mathrm{s}\right)$ for the $i^{\text {th }}$ tank. The volumetric inflow rate to tank 1 is assumed to be directly proportional to the applied pump voltage, such that (Eq. 2);

$F_{I N_{1}}=K_{p} V_{p}(t)$

where $K_{p}$ is a constant for the pump $\left(\mathrm{cm}^{3} / V s\right)$ and $V_{p}(t)$ is the voltage pump $(V)$. In addition, using Bernoulli's law, the outflow velocity from the orifice at the bottom of each tank is given by Eq. 3 .

$v_{\text {OUT }_{i}}(t)=\sqrt{2 g L_{i}(t)}$

( $g$ : the gravitational acceleration)

then, the outflow rate of each tank is calculated from Eq. 4;

$F_{\text {OUT }}(t)=a_{i} \sqrt{2 g L_{i}(t)}$

where $a_{i}$ is the cross-sectional area $\left(\mathrm{cm}^{2}\right)$ of the outflow orifice at the bottom of the $i$ th tank. The inflow of tank 2 is obtained as (Eq. 5);

$F_{I N_{2}}(t)=F_{O U T_{1}}(t)$

thus, using principle of mass balances and Eqs. 1-5, mathematical expression of the liquid level in the two tanks are obtained in Eq. 6 and Eq. 7.

$\dot{L}_{1}(t)=\frac{K_{p} V_{p}(t)-a_{1} \sqrt{2 g L_{1}(t)}}{A_{1}}$
$\dot{L}_{2}(t)=\frac{a_{1}}{A_{2}} \sqrt{2 g L_{1}(t)}-\frac{a_{2}}{A_{2}} \sqrt{2 g L_{2}(t) .}$

Using Eq. 6 at equilibrium $\left(\dot{L}_{1}(t)=0\right)$, the steadystate pump voltage $u_{1}(t)$, which produces the desired steady-state constant level $L_{10}$ in tank 1 and using Eq. 7 at equilibrium $\left(\dot{L}_{2}(t)=0\right)$, compute the steady-state level $L_{10}$ in Tank 1 that produces the desired steady-state constant level $L_{20}$ in Tank 2 are given by Eq. 8 and Eq. 9 .

$u_{1}(t)=a_{1} \frac{\sqrt{2 g L_{10}}}{K_{p}}$

$L_{10}=\left(\frac{a_{2}}{a_{1}}\right)^{2} L_{20}$

then, using the operating range corresponds to small departure voltages $\left(u_{1}(t)\right)$ and heights $\left(L_{1}(t), L_{2}(t)\right)$, one can define shifted variable as follows.

$l_{1}(t) \cong L_{1}(t)-L_{10}, l_{2}(t) \cong L_{2}(t)-L_{20}$

$u(t) \cong V_{p}(t)-u_{1}(t)$.

Hence the dynamic equations Eq. 6 and 7 can be converted as follows.

$\dot{l}_{1}(t)=-\frac{a_{1}}{A_{1}} \sqrt{2 g\left(l_{1}(t)+L_{10}\right)}+\frac{K_{p}}{A_{1}}\left(u(t)+u_{1}(t)\right)$

$\dot{l}_{2}(t)=-\frac{a_{1}}{A_{2}} \sqrt{2 g\left(l_{1}(t)+L_{10}\right)}-\frac{a_{2}}{A_{2}} \sqrt{2 g\left(l_{2}(t)+L_{20}\right)}$ 
The values of the parameters related to the two-

tank liquid level system are presented in Table 1.

Table 1: Parameters of two-tank liquid level system

\begin{tabular}{cccc}
\hline Physical Quantity & Symbol & Numerical Value & Units \\
\hline Tank 1, 2 diameters & $D_{1}, D_{2}$ & 4,5 & $\mathrm{~cm}$ \\
Tank 1 orifice diameters & $D_{o_{1}}$ & 0,4 & $\mathrm{~cm}$ \\
Tank 2 orifice diameters & $D_{o_{2}}$ & 0,4 & $\mathrm{~cm}$ \\
Pump constant & $K_{p}$ & 4,6 & $\mathrm{~cm}^{3} / \mathrm{Vs}$ \\
Gravitational constant & $g$ & 9,8 & $\mathrm{~cm}^{2} / \mathrm{s}^{2}$ \\
\hline
\end{tabular}

\section{Conventional feedforward (FFPI) controller design}

Conventional FFPI controllers are widely used in most industrial applications due to its simple tuning parameters and they can provide effective control performance of the linear systems. But the coupled tank system is nonlinear due to pump, valve characteristics and parameter variations. Therefore, the feedforward PI controller to the liquid level control system necessitates the linearization of the liquid level system model around an operating point. The linearized, liquid level system model and the feedforward PI controller are provided below. Linearize Eq. 10 and Eq. 11, about $\left(\mathrm{l}_{1}=0, \mathrm{l}_{2}=0, \mathrm{u}=\right.$ 0) to obtain;

$\dot{l}_{1}(t)=\alpha_{1} l_{1}(t)+\beta_{1} u(t)$

$i_{2}(t)=\alpha_{2} l_{2}(t)+\beta_{2} l_{1}(t)$

where

$\alpha_{1} \cong-\frac{a_{1}}{A_{1}} \sqrt{\frac{g}{2 L_{10}}}, \beta_{1} \cong \frac{K_{p}}{A_{1}}$

$\alpha_{2} \cong-\frac{a_{2}}{A_{2}} \sqrt{\frac{g}{2 L_{20}}}, \beta_{2} \cong \frac{a_{1}}{A_{2}} \sqrt{\frac{g}{2 L_{10}}}$

Next, the level control problem for Tank 2 (i.e., set point control of $\left.L_{2}(t)\right)$ is obtained via the subsystem decomposition of Eq. 12 and 13. $l_{2}$ and $l_{1}$ are addressed as the subsystem output and input in Eq. 13 , respectively. The transfer function models for the subsystem dynamics Eq. 12 and 13 are developed and Laplace transforms of these equations are taken in Eq. 15.

$G_{1}(s) \cong \frac{\beta_{1}}{s-\alpha_{1}}, G_{2}(s) \cong \frac{\beta_{2}}{s-\alpha_{2}}$

After that, the first PI controller for the Tank 1 subsystem and second PI controller for Tank 2 subsystem response have been designed according to percent overshoot and settling time values. The natural frequency and damping ratio characteristics of the two subsystems have been defined by using the percent overshoot and settling time requirements for the Tank 1 and 2 and the parameters of the controllers have been determined by natural frequency and damping ratio characteristics of the two subsystems. The PI controllers have been given by Eq. 16 .

$C_{k}(s)=K_{P k}+\frac{K_{I k}}{s}, k=1,2$
The block diagrams of Feedforward PI Control Loop for Tank-1 and Tank-2 have been shown in Figs. 2 and 3.

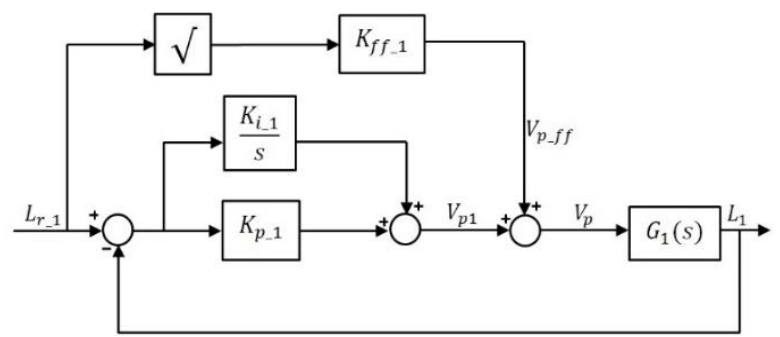

Fig. 2: Tank 1 water level feedforward PI control loop

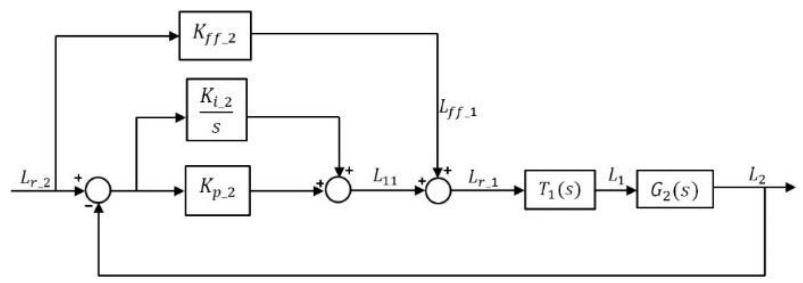

Fig. 3: Tank 2 water level feedforward PI control loop

As it can be seen in Figs. 3 and 4, the feedforward action is necessary since the PI control system is designed to compensate for small variations (disturbances) from the linearized operating points $\left(V_{p 0}, L_{10}, L_{20}\right)$. In other words, while the feedforward action compensates for the water withdrawal (due to gravity) through tank 1 and 2 bottom outlet orifice, the PI controller compensates for dynamic disturbances.

\section{Fractional controller design}

The fractional-order $\mathrm{PI}^{\lambda} \mathrm{D}^{\mu}$ controller is a generalization of the integer-order PID controller exploiting the richness offered by the noninteger orders of the Laplace variable $s$. Denoting $C_{c}(s)$ as the transfer function associated to it, an analog $\mathrm{PI}^{\lambda} \mathrm{D}^{\mu}$ controller is described as given in the following:

$C_{c}(s)=K_{p}+K_{i} s^{-\lambda}+K_{d} s^{\mu},(\lambda, \mu>0)$

where $K_{p}$ is the proportional constant, $K_{i}$ is the integration constant, and $K_{d}$ is the differentiation constant. Clearly, for $\lambda=1$ and $\mu=1$, controller (17) takes the classical PID form.

The performance of the $\mathrm{PI}^{\lambda} \mathrm{D}^{\mu}$ controller for the control of the considered system is expected to be better than that of the classical PID controller due to its higher degrees of freedom for tuning.

On the other hand, the dynamic behavior of fractional transfer functions can be classified into 
two subsections, either integer or digital transfer functions. The usefulness of such approximations may be summarized as follows:

1) While there are numerical methods to solve fractional differential equations, methods for integer differential equations are better known and are the ones widely available in commercial software.

2) While hardware implementations of fractional controllers are possible, it is often easier and cheaper to implement in hardware integer transfer functions only.

The fractional-order PID controller should ensure that the given gain crossover frequency and the phase margin are achieved and the phase derivative w.r.t. the frequency is zero, i.e., the phase Bode plot is flat, at the gain crossover frequency so that the closed-loop system is robust to gain variations and the step response exhibits an iso-damping property.

The block diagram of fractional-order PID control loop for Tank-1 and Tank-2 has been shown in Fig. 4.

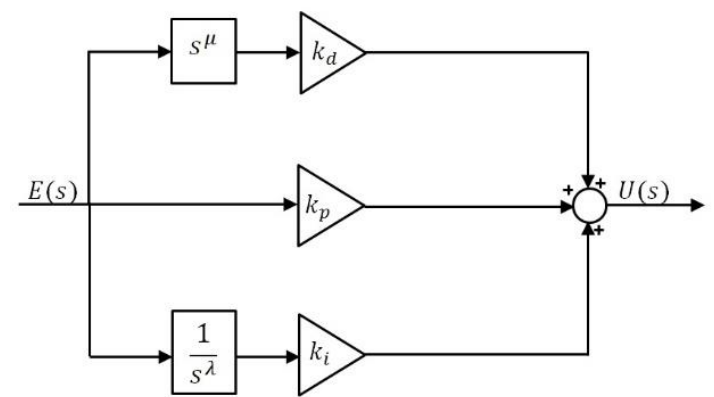

Fig. 4: The block diagram of fractional-order PID control loop

We will design the controller which gives us a step response of the feedback control loop independent of payload changes (iso-damping). In the frequency domain point of view, it means the phase margin independent of the disturbance changes.

The phase margin of the controlled system is;

$\Phi_{m}=\arg \left[C_{c_{1,2}}\left(j \omega_{g}\right) G_{1,2}\left(j \omega_{g}\right)\right]+\pi$

As given in (Podlubny, 1999b; Roy and Roy, 2016), with regard to the general characteristics of Bode's ideal transfer function, the desired phase margin is can be obtained for the considered system. With these situation, we obtain fractional $I^{\lambda} D^{\mu}$ controllers, which is a particular case of the $\mathrm{PI}^{\lambda} \mathrm{D}^{\mu}$ controllers for the tank 1 and tank 2 control as follow;

$$
C_{c}(s)=K_{p}+K_{i} s^{-\lambda}+K_{d} s^{\mu},(\lambda, \mu>0)
$$

As can be seen from (19), the first term is a differentiator, and the second term of the equation is an integrator. $K_{i}$ and $K_{d}$ values are calculated from (18), and the $K_{p}$ value is also considered as 1.126 for the tank 1 and 2.256 for the tank 2 control.

\section{Experimental results}

The fractional-order control was implemented on an experimental, two-tank, liquid level system. The experimental state coupled, two tanks liquid level control system is shown in Fig. 5. The controller has been implemented using SIMULINK 2014 from MathWorks running on a personal computer with Intel Core $\mathrm{i} 7-47903.6 \mathrm{GHz}$ processor. The experiments have been realized using the Q2 USB data acquisition device from Quanser which is ideal for rapid control prototyping and delivers superior real-time performance.

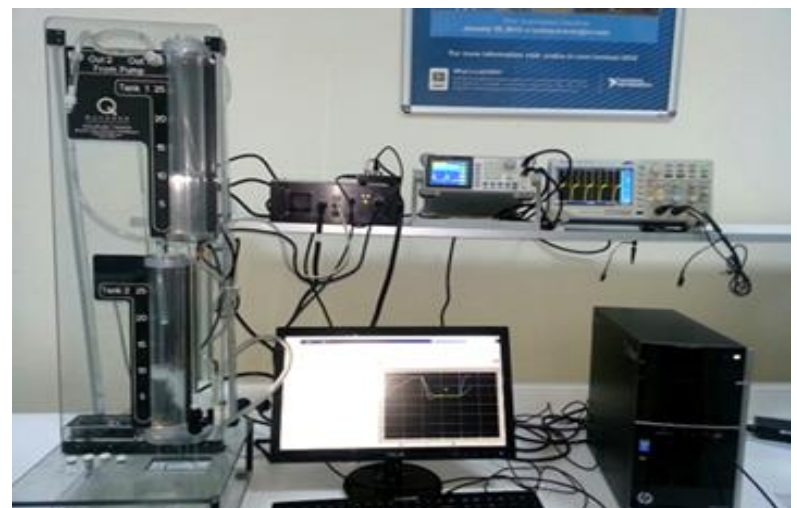

Fig. 5: The experimental state coupled, two tanks liquid level control system

In this section, experimental results have been presented to show the performance of the feedforward PI and fractional-order $\mathrm{PI}^{\lambda} \mathrm{D}^{\mu}$ controllers for both single and two tank level control system. The steady state constant level of tank 1 and tank 2 has been adjusted as $L_{10}=17, L_{20}=17 \mathrm{~cm}$ for all experiments. In the tank 1 and tank 2 configuration experiments, the performance of the both controllers has been investigated for the desired square trajectories, respectively. The reference of the square wave is crucial since the controlled system may have a sudden rising and reducing liquid levels in a period.

An experimental process can be divided into two sections;

(i) Feedforward PI controller: Taking into account the conventional controller design, the level in tank 1 should satisfy the following design performance requirements;

1. Operating level tank 1 at $17 \mathrm{~cm}$

2. Percent overshoot equal to 0 (No overshoot)

3. $\% 2$ settling time equal to 5 seconds

4. No steady-state error.

Hence the control parameters are illustrated for tank 1 configuration as below;

$K_{p_{1}}=7.24, K_{i_{1}}=3.36, K_{f f_{1}}=2.39$

The level in tank 2 should satisfy the following design performance requirements;

1. Operating level tank 2 at $17 \mathrm{~cm}$ 
2. Percent overshoot equal to 0 (No overshoot)

3. $\% 2$ settling time equal to 20 seconds

4. No steady-state error.

Hence the control parameters are illustrated for tank 2 configuration as below;

$K_{p_{2}}=5.49, K_{i_{2}}=0.69, K_{f f_{2}}=1$

Figs. 6 and 7 present the tracking response for the tank 1 and tank 2 configuration using conventional feedforward PI controller.

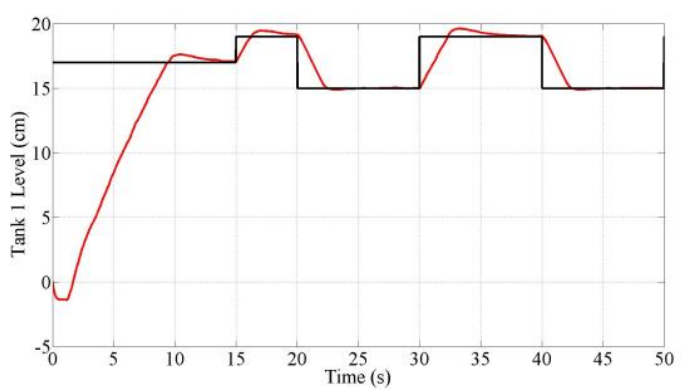

Fig. 6: The conventional feedforward PI controller for Tank 1 configuration

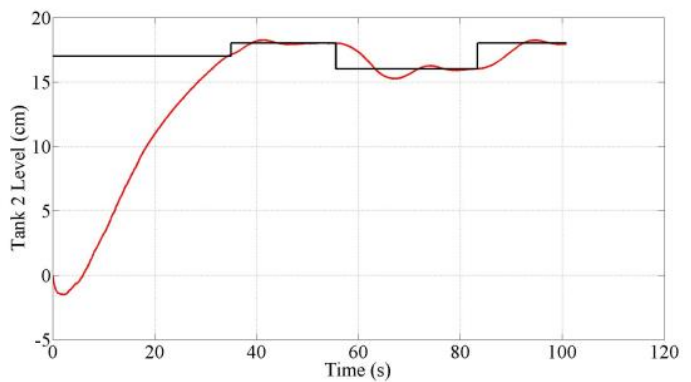

Fig. 7: The conventional feedforward PI controller for Tank 2 configuration

(ii) Fractional-order $\mathrm{PI}^{\lambda} \mathrm{D}^{\mu}$ controllers: Controller output in Eq. 19 which is obtained based on the Eq. 18 , has been performed in real time with the coupled tank system. And the outcome of the tracking response for the desired trajectories is shown in Figs. 8 and 9.

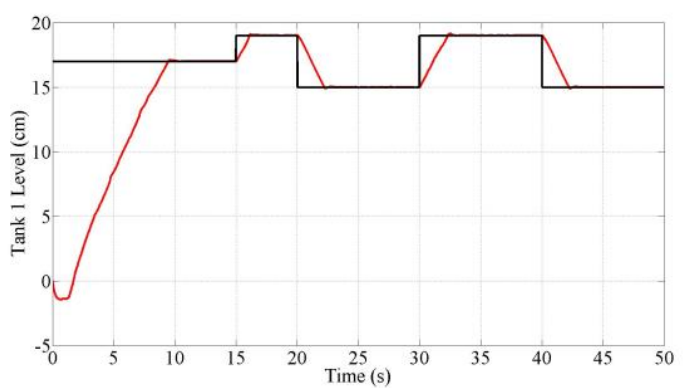

Fig. 8: The fractional-order $\mathrm{PI}^{\lambda} \mathrm{D}^{\mu}$ controller for Tank 1 configuration

As can be seen from the Figs. 6, 7, 8, and 9, overshoot values are almost eliminated for the fractional-order $\mathrm{PI}^{\lambda} \mathrm{D}^{\mu}$ controller case in tank 1 and tank 2 configurations, respectively. Additionally, both transient and steady-state error values are decreased by utilizing the fractional-order $\mathrm{PI}^{\lambda} \mathrm{D}^{\mu}$ controller.

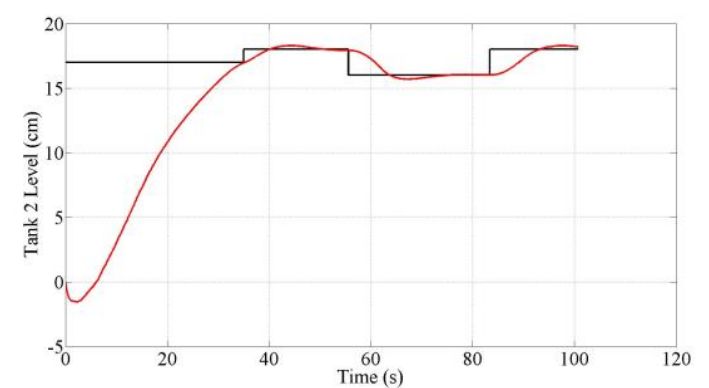

Fig. 9: The fractional-order $\mathrm{PI}^{\lambda} \mathrm{D}^{\mu}$ controller for Tank 2 configuration

In order to demonstrate the efficiency of the proposed controller, the mean squared error (MSE) values with respect to level values of tank 1 and tank 2 are calculated and tabulated in Table 2 for both cases. As it is well known that; the mean squared error (MSE) value can also be used to illustrate the performance of the proposed controller. For this reason, MSE values are also tabulated in Table 2 as another performance criterion.

Table 2: The MSE values

\begin{tabular}{ccc}
\hline & $\begin{array}{c}\text { Tank 1 } \\
\text { Configuration }\end{array}$ & $\begin{array}{c}\text { Tank 2 } \\
\text { Configuration }\end{array}$ \\
\cline { 2 - 3 } $\begin{array}{c}\text { FF-PI controller } \\
\text { Fractional-order PI }{ }^{\lambda} \mathrm{D}^{\mu}\end{array}$ & 2.07 & 9.8 \\
controller & 1.55 & 8.9 \\
\hline
\end{tabular}

As tabulated in Table 2, the MSE value of the FFPI is greater than that of the fractional-order $\mathrm{PI}^{\lambda} \mathrm{D}^{\mu}$ controller.

\section{Conclusion}

This paper used the fractional-order control techniques in order to develop accuracy of the liquid level tracking in coupled level two tank system. Firstly, in order to investigate the effects of this system dynamics and control technique, dynamic model of the system has been developed. And then, the conventional feedforward PI controller has been applied to the real time system. Finally, fractionalorder control technique has been used for tank 1 and tank 2 configuration. It is observed that not only transient but also steady-state error values have been reduced with the aid of the fractional-order controller for tracking control purpose.

According to the MSE values between the desired and obtained values, obtained results for the fractional-order $\mathrm{PI}^{\lambda} \mathrm{D}^{\mu}$ controller are more superior to that of the optimally tuned PID.

In future, we would like to design an observer based on more complex controller structure for the considered system. With the aid of the observer, it is expected that the error value for desired and obtained cases will be reduced.

\section{References}

Altet O, Moreau X, Moze M, Lanusse P, and Oustaloup A (2004). Principles and synthesis of hydractive CRONE suspension. Nonlinear Dynamics, 38(1): 435-459. 
Bhuvaneswari NS, Uman G, and Rangaswamy TR (2009). Adaptive and optimal control of a non-linear process using intelligent controllers. Applied Soft Computing, 9(1): 182-190.

Boiocchi R, Mauricio-Iglesias M, Vangsgaard AK, Gernaey KV, and Sin G (2015). Aeration control by monitoring the microbiological activity using fuzzy logic diagnosis and control - Application to a complete autotrophic nitrogen removal reactor. Journal of Process Control, 30: 22-33.

Derdiyok A and Başçi A (2013). The application of chattering-free sliding mode controller in coupled tank liquid-level control system. Korean Journal of Chemical Engineering, 30(3): 540 545.

Domingues J, Valerio D, and Costa J (2010). Rule-based fractional control of an irrigation canal. Journal of Computational and Nonlinear Dynamics, 6(2): 1-6.

Dumlu A and Erenturk K (2014). Trajectory tracking control for a 3-DOF parallel manipulator using fractional-order $\mathrm{PI}^{\lambda} \mathrm{D}^{\mu}$ control. IEEE Transactions on Industrial Electronics, 61(7): 3417-3426.

Kaplanoglu E, Arsan T, and Varol HS (2015). Predictive control of a constrained pressure and level system. Turkish Journal of Electrical Engineering and Computer Sciences, 23(3): 641 655.
Musmade BB and Patre BM (2013). Feedforward-plus-sliding mode controller design with experimental application of coupled tank system. Transactions of the Institute of Measurement and Control, 35(8): 1058-1067.

Oustaloup A, Mathieu B, and Lanusse P (1995). The CRONE control of resonant plants: application to a flexible transmission. European Journal of Control, 1(2): 113-121.

Podlubny I (1999a). Fractional differential equations: An introduction to fractional derivatives, fractional differential equations, to methods of their solution and some of their applications. Academic Press, New York, USA.

Podlubny I (1999b). Fractional-order systems and $\mathrm{PI}^{\lambda} \mathrm{D}^{\mu}-$ controllers. IEEE Transactions on Automatic Control, 40(1): 208-214.

Qi W, Liu J, and Christofides PD (2011). A distributed control framework for smart grid development: Energy/water system optimal operation and electric grid integration. Journal of Process Control, 21(10): 1504-1516.

Roy P and Roy BK (2016). Fractional order PI control applied to level control in coupled two tank MIMO system with experimental validation. Control Engineering Practice, 48: 119-135. 